\title{
Quantification of primary frequency control provision from battery energy storage systems connected to active distribution networks
}

\author{
Giacomo - Piero Schiapparelli, Stefano Massucco \\ DITEN, University of Genova, Genova, Italy \\ schiapparelli.g@gmail.com, stefano.massucco@unige.it
}

\author{
Emil Namor, Fabrizio Sossan, Rachid Cherkaoui, \\ Mario Paolone \\ DESL, EPFL, Lausanne, Switzerland \\ \{emil.namor, fabrizio.sossan, rachid.cherkaoui, \\ mario.paolone\}@epfl.ch
}

\begin{abstract}
In this paper, we consider the provision of primary frequency control by using battery energy storage systems (BESSs). In particular, we use a standard droop-based frequency control for a BESS where the control action (i.e. the BESS power output) consists in the contribution of two additive terms: the regulating power, proportional to the frequency deviations, and an offset term computed to manage the BESS State-of-Energy (SOE). In the context of such a control scheme, we propose a method to forecast the BESS energy for regulation needs and we show that the inclusion of such a forecast can increase the regulating power provision. Finally, we demonstrate the performance of the proposed approach by means of a real-scale experimental setup composed by a grid-connected $720 \mathrm{kVA} / 560 \mathrm{kWh}$ BESS installed at the EPFL campus in Lausanne, Switzerland.
\end{abstract}

Index Terms-Battery energy storage systems, primary frequency regulation, control, forecast.

\section{INTRODUCTION}

There is a general technical consensus that the progressive displacement of conventional generation in favour of production from renewable sources requires distributed generation (DG) and storage to provide grid ancillary services. In this context, grid-connected battery energy storage systems (BESSs) are gaining increasing focus thanks to their fast ramping compared to conventional generation units [1]-[3]. Several authors have proposed different methods to provide primary frequency regulation (PFR) with BESSs, e.g. by using adaptive droop control [4], offset frequency control signals [5] and model predictive control (MPC) exploiting shortterm predictions of the grud frequency [6]. In this paper, we consider the problem of the provision of PFR with BESSs using a droop-based control strategy. The control is composed by three consecutive phases: $i$ ) the a-priori computation of the droop coefficient with an optimisation problem; ii) the periodical (with a period of length $T$, ranging from 1 to 24 hours), computation of an offset power to maintain continuous operation, based on the BESS state of energy (SOE) and forecast of the energy needed for regulation via autoregressive (AR) models; iii) the real-time control, which implements a power setpoint consisting in the sum of the regulating and offset power.
With regard to this general control structure we: $i$ ) introduce two forecasting methods to predict the energy needed for regulation over subsequent periods by using AR models of different orders; ii) compute the maximum possible regulating capacity according to technical constraints and desired reliability requirements; iii) examine how the duration of the period for the computation of both the droop coefficient and the power offset affect the performance of such control scheme and $i v$ ) validate the method by simulations and experiments with a grid-connected BESS.

An extensive comparison between the two forecasting methods is carried out to assess the benefits of incorporating the forecast of the regulating energy need in the proposed control. The performance of the proposed control framework is validated by simulations in Matlab/Simulink and experimentally tested using a grid-connected $720 \mathrm{kVA} / 560 \mathrm{kWh}$ BESS installed at the EPFL campus in Lausanne, Switzerland [7].

The contributions of this paper are twofold. First, we introduce a method to forecast the BESS energy need due to PFR and we demonstrate that it allows to increase the provision of regulating power. Second, we perform an extensive experimental campaign to: $i$ ) evaluate the reliability and success rate of the control framework; ii) evaluate the ability of the BESS to follow the regulation signal, with performance metrics as in [8]; iii) infer the long-term performance of the proposed method using the above mentioned simulation environment.

The rest of the paper is structured as follows. In Section II, we describe the methods to forecast the energy need of a BESS performing PFR. In Section III, we describe the implemented control algorithms. In Section IV, the main simulation and experimental results are presented. Section V summarizes the main contributions and perspectives of this paper.

\section{FORECAST OF ENERGY REQUIRED FOR REGULATION}

Since BESSs can store a limited amount of energy, in order to provide a given amount of regulating power for a predetermined duration, a BESS needs to continuously adopt its reservoir level. Therefore, in order to maximise the capacity of providing regulating power, it is important to quantify the energy that the BESS needs to make available to accomplish 
the PFR service provision over a future period of duration period $T$. Since regulating power $P_{p f r}$ is proportional to frequency deviations $\Delta f$ from the nominal frequency, the regulating energy $E_{p f r}$ is proportional to the integral of such deviations over the considered interval:

$$
E_{p f r}=\int_{T} P_{p f r} d t=\int_{T} \alpha \Delta f d t=\alpha \int_{T} \Delta f d t=\alpha W_{f},
$$

where $W_{f}$ is the integral over a time window $T$ of the deviations of frequency from its nominal value, and $\alpha$ is the droop coefficient in $\mathrm{kW} \mathrm{Hz}^{-1}$. It is worth noting that (1) assumes that the regulating power is also deployed in response to frequency deviations within $\pm 10 \mathrm{mHz}$. Typically, European grid codes do not require activation within this band [9]. However, it can be demonstrated that the disregard of such practice has a negligible effect on the resulting $W_{f}$ values and on the subsequent control.

The quantity $W_{f}$, over a long enough time horizon is equal to zero (considering a power grid with a sufficient amount of secondary frequency control reserve). However, this is not generally true for shorter intervals, and we show that it is possible to forecast the value of $W_{f}$ and use it to achieve efficient PFR strategies. The analysis detailed in these paragraphs has been carried out on two separate datasets, respectively composed by two years (2014-2015) of frequency measurements collected by National Grid Electricity Transmission (NGET) ${ }^{1}$ and by one year (2016) of on-site frequency measurements at EPFL [10]. From the frequency timeseries, we have computed several $W_{f}$ timeseries, with integration intervals $T$ of increasing length from 1 to 24 hours. The objective is to verify the possibility to use autoregressive models (AR) in order to forecast $W_{f}$.

The usage of AR models requires the analysis of the probability density function of the variable $W_{f}$ and its autocorrelation [11]. Figure 1 shows in red the quantile-quantile plots obtained for two $W_{f}$ timeseries (respectively from the NGET and EPFL databases and with different $T$ ) and in blue the same plot for the quantiles of a normal distribution. Such plots demonstrate that the data of such timeseries are close to normally distributed and suggest the use of AR models.

Figure 2-a shows the autocorrelation of the timeseries composed by subsequent $W_{f}$ values computed for the NGET database and with $T=1 \mathrm{~h}$ as an example. Modelling such timeseries as a persistent process (i.e. assuming that there are not relevant dynamics that can be captured), at every timestep we predict the value $\widehat{W}_{f}(k)=\mu_{f}=0$. For such model (hereafter $\mathrm{AR}(0))$, the model residual corresponds the value of $W_{f}$ itself, being $r_{k}=W_{f}(k)-\widehat{W}_{f}(k)=W_{f}$. We see that the residuals for such model present an autocorrelation that is, for the lower lags, higher than for white noise (indicated by the blue lines in the plot). This suggests that $W_{f}$ contains dynamics that can be modelled with an higher order model. Figure 2-b shows the autocorrelation of the model residuals obtained, for the same dataset, with an autoregressive model of order 8 (i.e.

\footnotetext{
${ }^{1}$ Dataset downloaded from http://www2.nationalgrid.com/EnhancedFrequency-Response.aspx on April 24 $4^{\text {th }}, 2017$.
}
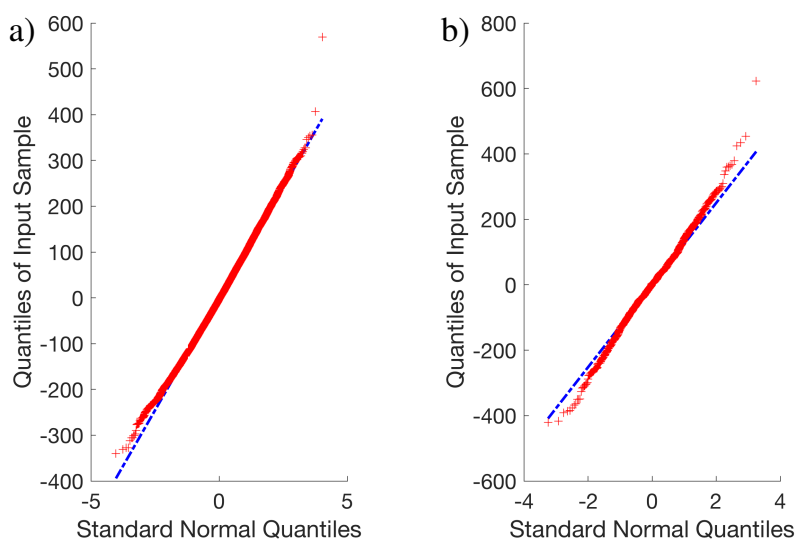

Figure 1. Q-Q plot of the residuals of $W_{f}$ (red) versus the normal standard (blue) built using a) $T=1 \mathrm{~h}$, data from the NGET database and b) $T=6 h$, data from EPFL database.
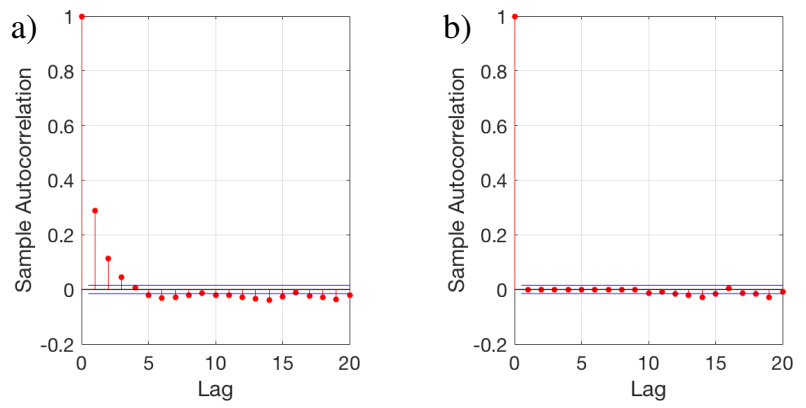

Figure 2. Autocorrelation function of the residuals of a) $A R(0)$ and b) $A R(8)$, both computed with $T=1 \mathrm{~h}$ and data from the NGET database.

the lowest order that successfully captures all the relevant dynamics for all the period lengths $T$ ), hereafter referred as $\mathrm{AR}(8)$. It can be seen that the residual autocorrelation does not show, in this case, any value outside the band identifying white noise. Similar results are obtained for all values of $T$ and for both datasets considered (not reported here for sake of space). Table I compares the standard deviation of the residuals of models $\mathrm{AR}(0)$ and $\mathrm{AR}(8)$ obtained for integration periods $T$ of increasing duration. It can be observed that this value is lower for the $\mathrm{AR}(8)$ model for all $T$.

TABLE I

RESIDUAL STANDARD DEVIATION $\sigma$ FOR AR(0) AND AR(8), DATA FROM NGET DATABASE.

\begin{tabular}{|c|ccccccc|}
\hline$T[h]$ & 1 & 2 & 3 & 4 & 6 & 12 & 24 \\
\hline$A R(0)$ & 96 & 154 & 201 & 243 & 312 & 428 & 575 \\
\hline$A R(8)$ & 92 & 150 & 197 & 239 & 307 & 408 & 516 \\
\hline
\end{tabular}

Using such a prediction model provides two relevant results for the PFR control strategy. First, a model with lower variance allows to use a higher droop coefficient and therefore provide higher regulating power (as detailed in Section III-A). Second, the predicted value $\widehat{W}_{f}$, recomputed periodically, can be used to improve the state of energy management associated to such droop coefficient (as detailed in Section III-B). 


\section{CONTROL STRATEGY}

The BESS control consists in a standard droop-based frequency regulation strategy where the BESS's active power output is given by the sum of the regulating power $P_{f}$ and the offset term $P_{S O E}$, computed periodically to keep the SOE within its technical bounds. For each period, the control is designed to restore the reference condition of State-of-Energy, $S O E_{0}$. It acts in three stages:

1) a-priori computation of the droop coefficient $\alpha$ given by the solution of an optimization problem. The latter takes into account the statistical properties of $W_{f}$ to determine the maximum value of $\alpha$ (hereafter $\alpha_{\max }$ ) that can be used for periods of the given duration $T$. The resulting $\alpha_{\max }$ is then fixed for all considered periods $T$ or, in the context of ancillary service markets, fixed within the time horizon for the bidding of the frequency regulation service (e.g. one week).

2) the period-ahead operation where, on the basis of the forecast of the energy required for one period of operation $T$ and the knowledge of the SOE of the BESS, the offset term $P_{S O E}$ for such period is computed. This task is defined hereafter as State-of-Energy Management (SOE-M) and is repeated for each i-th period $T_{i}$;

3 ) the real-time control, where the regulating action is actuated with 1 second resolution.

Figure 3 shows the control diagram.

\section{A. Droop coefficient computation}

The BESS SOE is defined as the integral over the time of the power output of the battery ${ }^{2}$ :

$$
\begin{aligned}
\operatorname{SOE}\left(T_{i}+t\right) & =\frac{1}{E_{n}} \cdot \int_{T_{i}}^{T_{i}+t} P(\tau) d \tau+\operatorname{SOE}\left(T_{i}\right) \\
& =\frac{\alpha}{E_{n}} \cdot \int_{T_{i}}^{T_{i}+t} \Delta f(\tau) d \tau+\operatorname{SOE}\left(T_{i}\right),
\end{aligned}
$$

where $E_{n}$ is the BESS nominal energy capacity. The value of the droop coefficient $\alpha$ is determined by solving the optimisation problem detailed below, aiming at maximizing the droop coefficient while respecting the constraints on the energy that can be stored in the battery. The optimization constraints are built to ensure a continuous operation of the battery. Starting from an initial condition of $S O E\left(T_{i}\right)=S O E_{0}$, the maximum deviation during one period $T$ must be equal to $S O E_{0}$, thus, $0 \leq S O E\left(T_{i+1}\right) \leq 2 S O E_{0}$. Therefore, since $\operatorname{SOE}\left(T_{i+1}\right) \in\{0,1\}$, the reference condition $S O E_{0}$ has to be chosen in the range $[0,0.5]$. From equation (3), SOE $\left(T_{i+1}\right)$ can be expressed as function of $W_{f}$ :

$$
\operatorname{SOE}\left(T_{i+1}\right)=\frac{\alpha \cdot W_{f}}{E_{n}}+\operatorname{SOE}\left(T_{i}\right) .
$$

Therefore, considering a confidence level $\rho$ and the value distribution of the residuals of the energy need forecasting

\footnotetext{
${ }^{2}$ At this stage we are neglecting the battery losses. In Section IV-B we will show that these have a negligible impact on the computation of $\alpha$ and that the $\triangle S O E$ due to the battery losses can be absorbed by the offset power computed for the subsequent period $T_{i+1}$.
}

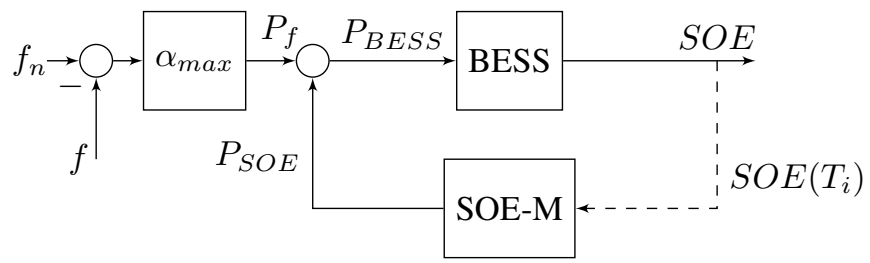

Figure 3. Block diagram of the proposed method to operate the PFR.

as defined in section II, the optimization problem can be expressed as:

$$
\alpha_{\max }=\underset{\alpha}{\arg \max }\{\alpha\}
$$

subject to:

$$
\begin{aligned}
& \operatorname{SOE}\left(T_{i+1}\right)=\frac{\alpha \cdot W_{f}}{E_{n}}+\operatorname{SOE}\left(T_{i}\right) \\
& \operatorname{Pr}\left(\left|\operatorname{SOE}\left(T_{i+1}\right)-\operatorname{SOE}\left(T_{i}\right)\right| \leq S O E_{0}\right) \geq \rho
\end{aligned}
$$

where $\operatorname{Pr}(\cdot)$ denote the probability that the argument occurs. This problem has an analytical solution. By applying (4), (7) can be written as:

$$
\operatorname{Pr}\left(\left|W_{f}\right| \leq \frac{S O E_{0} E_{n}}{\alpha}\right) \geq \rho .
$$

Since $W_{f}$ is a normally distributed stochastic process with variance $\sigma_{f}$ (as shown in Section II), Eq. 8 is satisfied by:

$$
\alpha \leq \frac{S O E_{0} \cdot E_{n}}{k \cdot \sigma_{f}}
$$

where the value of $k$ is related to that of the selected $\rho$, e.g. $k=1.96$ for $\rho=0.95, k=2.58$ for $\rho=0.99$, etc..

Since the objective in 5 is to maximise the regulating power provided with the BESS, the maximum droop coefficient and solution of the problem 5-7 is:

$$
\alpha_{\max }=\frac{E_{n} \cdot S O E_{0}}{k \cdot \sigma_{f}} .
$$

The value of the standard deviation of the residuals $\sigma_{f}$ depends on the autoregressive model used and length of the period $T$. An autoregressive model of higher order, such as $\operatorname{AR}(8)$ has a lower variance of the residuals obtained with an $\mathrm{AR}(0)$ model. Therefore, its use is convenient as it leads to a higher value for $\alpha$ and a higher regulating capacity.

\section{B. State-of-Energy Management}

The SOE-M uses a power offset profile to restore the initial condition, $S O E_{0}$, at the beginning of each consecutive operation period in order to ensure a reliable and continuous regulation. At the beginning of the period, the SOE-M measures the SOE of the battery, forecasts the evolution of the SOE in the next period and, on the basis of this result, computes the offset power profile. The offset power is computed to avoid hitting the SOE limits in the upcoming period of operation. To achieve this goal, the prediction of the SOE variation due to regulation at the end of the upcoming period, which is provided by the forecasting methods detailed in section II, 
is exploited. When using the $\mathrm{AR}(0)$ model, the predicted $\mathrm{SOE}$ at the end of the upcoming period of operation, $\widehat{\operatorname{SOE}}\left(T_{i+1}\right)$, is equal to the current one $\widehat{S O E}\left(T_{i}\right)$. An autoregressive model of higher order, such as $\operatorname{AR}(8)$, allows for a more precise prediction and $\widehat{S O E}\left(T_{i+1}\right)$ will differ, in general, from $\widehat{S O E}\left(T_{i}\right)$. The energy to be exchanged by the BESS in the following period will be therefore:

$$
\widehat{E}_{S O E}=\left[S O E_{0}-\widehat{S O E}\left(T_{i+1}\right)\right] E_{n},
$$

where:

$$
\begin{aligned}
& \widehat{S O E}\left(T_{i+1}\right)=\widehat{S O E}\left(T_{i}\right) \quad \text { for } \operatorname{AR}(0) \text {, } \\
& \widehat{S O E}\left(T_{i+1}\right)=\widehat{S O E}\left(T_{i}\right)+\triangle \widehat{S O E} \widehat{S F}_{i} \text { for } \operatorname{AR}(8) \text {. }
\end{aligned}
$$

The offset power to satisfy such energy need is set to be constant over the period $T_{i+1}-T_{i}$, and equal to:

$$
P_{S O E}^{i}=\frac{\left[S O E_{0}-\widehat{S O E}\left(T_{i+1}\right)\right] \cdot E_{n}}{T_{i+1}-T_{i}}
$$
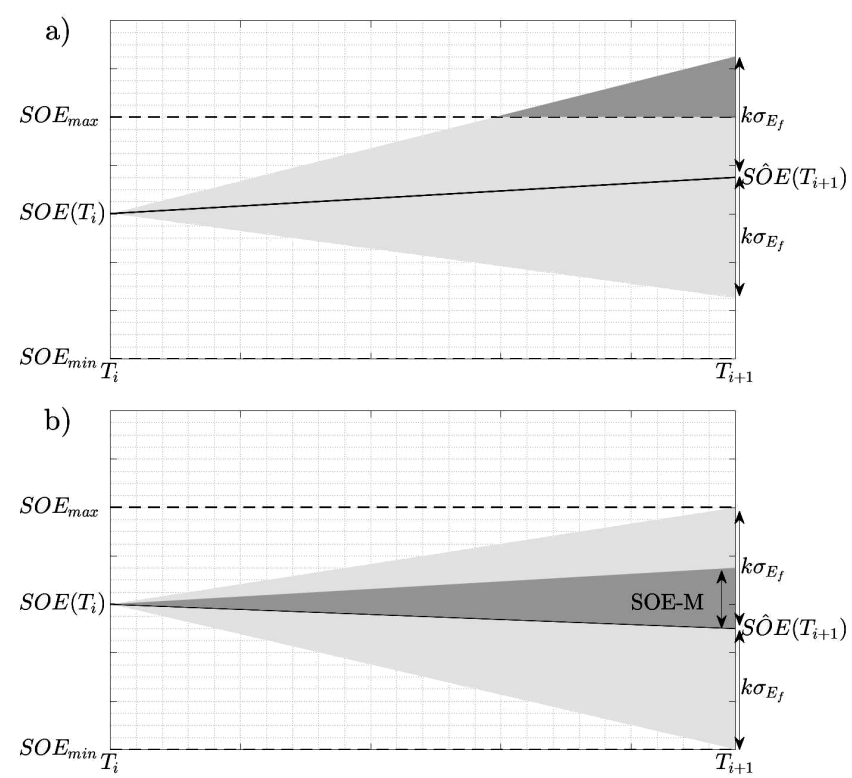

Figure 4. Expected trajectories of the energy stored in the BESS over a period $T$ a) without and $b$ ) with the action of the SOE-M.

The impact of the offset profile is shown graphically in Figure 4. At the beginning of the period the state of energy is in principle different from $S O E_{0}$. Moreover, the expected $\mathrm{SOE}$ at the end of the period will vary of a quantity $\triangle \widehat{S O E}_{i}$, predicted via the models proposed in Section II. The space of the expected trajectories for the upcoming period therefore is tilted by a quantity which depends on the value of $S O E\left(T_{i}\right)$ and $\triangle \widehat{S O E}_{i}$. At the end of the period, the state of energy is expected to be in the range $\left.\left[\widehat{S O E}\left(T_{i+1}\right) \pm k \cdot \sigma_{E_{f}}\right)\right]$. Such interval may exceed the range of capable $S O E$, and so the reliability of the PFR provision can not be ensured (see Figure 4-a).
When the offset profile is implemented, the space of the expected trajectories is modified. The offset power is constant over all the period, so the energy injected or extracted form the battery during such period follows a linear increasing or decreasing shape,

$$
S O E_{S O E}^{i}(t)=\frac{1}{E_{n}} \int_{0}^{t} P_{S O E}^{i}(\tau) d \tau=\frac{P_{S O E}^{i}}{E_{n}} \cdot t .
$$

therefore, the expression of the evolution of the state of energy during the time can be written as:

$$
\begin{aligned}
\operatorname{SOE}\left(T_{i}+t\right) & =\frac{1}{E_{n}} \int_{T_{i}}^{T_{i}+t}\left[ \pm P_{S O E}^{i}(\tau)+P_{f}(\tau)\right] d \tau+\operatorname{SOE}\left(T_{i}\right) \\
& =+\frac{P_{S O E}^{i}}{E_{n}} \cdot t+\alpha \frac{W_{f}(t)}{E_{n}}+\operatorname{SOE}\left(T_{i}\right)
\end{aligned}
$$

The addition of the power offset profile impacts the evolution of the state of energy by aligning the confidence interval on SOE $\left(T_{i+1}\right)$ with the BESS capability. From the graphical point of view, these relationships can be interpreted as in figure 4-b. Applying a constant power offset, the impact on the evolution of the SOE consist in a ramp added to the state of energy trajectory. The space of the possible trajectories is therefore shifted and the confidence level, at the end of the following period, results to be centered within the capability of the BESS.

\section{Real-time control}

In real-time, the frequency deviation $\Delta f$ is measured with 1 second resolution and every second the BESS power setpoint is refreshed as:

$$
P_{B E S S}=\Delta f \cdot \alpha+P_{S O E} .
$$

Where the value of $\alpha_{\max }$ is determined a priori as in subsection III-A and the value of $P_{S O E}$ is computed as described in Section III-B and updated once every period $T$.

\section{RESUlts}

In this Section, the main results obtained by applying the proposed methods are shown. Notably, Subsection IV-A shows the values of droop coefficients determined for the two datasets, the two forecasting models and for various values of $T$. Subsection IV-B shows the simulation results obtained for such values and compares the performances of the control based on $\mathrm{AR}(0)$ and on $\mathrm{AR}(8)$. Finally, Subsection IV-C shows results obtained applying the proposed methods to the control of a $720 \mathrm{kVA} / 560 \mathrm{kWh}$ grid-connected BESS. These are with the objective of validating experimentally the proposed methods, evaluating the BESS performances in performing PFR and validating the simulation environment used in Subsection IV-B. 


\section{A. Droop coefficient computation}

The algorithm for the computation of the droop coefficient $\alpha_{\max }$ has been used for each of the considered periods. Tables II and III show such results. Notably, Table II refers to the droop coefficient values computed from the frequency dataset provided by NGET. Table III, refers to the data collected in one year at the EPFL campus and that have been used in the experiments described in Section IV-C.

The method presented in Section III-A determines the value of $\alpha_{\max }$ on the basis of the energy capability of the BESS. In the case of the EPFL $720 \mathrm{kVA} / 560 \mathrm{kWh}$ BESS, since both energy and power ratings are given, also the latter should be used to determine the $\alpha_{\max }$. The theoretical value of $\alpha_{\max }$ corresponds to the minimum value between $\alpha_{\max }^{e}$ (i.e. the maximum droop coefficient based on the BESS energy capability, found as in Section III-A) and $\alpha_{\max }^{p}$ (i.e. the maximum droop coefficient compatible with the BESS power ratings). The latter term can be computed as follows:

$$
\begin{aligned}
P_{S O E}^{\max } & =\frac{S O E_{0} \cdot E_{n}}{T_{i+1}-T_{i}}, \\
P_{f}^{\max } & =P_{0}-P_{S O E}^{\max }, \\
\alpha_{\text {max }}^{p} & =\frac{P_{f}^{\max }}{\Delta f^{\max }},
\end{aligned}
$$

where $P_{S O E}^{\max }$ is the maximum possible value of the power offset, $P_{0}$ is the rated power of the BESS, and $P_{f}^{\max }$ is the maximum regulating power. All the values in Table III have been computed considering a grid-connected $720 \mathrm{kVA} / 560 \mathrm{kWh}$ BESS and the activation of full reserve power for frequency deviation beyond $\pm 200 \mathrm{mHz}$, as dictated by ENTSO-E grid codes [12].

TABLE II

DROOP COEFFICIENT VALUES $[k W / H z]$, NGET DATABASE.

\begin{tabular}{|c|c|ccccccc|}
\hline$T[h]$ & $\rho$ & 1 & 2 & 3 & 4 & 6 & 12 & 24 \\
\hline \multicolumn{7}{|c|}{ AR(0) model } \\
\hline$\alpha_{\max }^{e}$ & $95 \%$ & 5325 & 3321 & 2555 & 2115 & 1646 & 1201 & 893 \\
$\alpha_{\max }^{e}$ & $99 \%$ & 4046 & 2523 & 1941 & 1607 & 1251 & 913 & 679 \\
\hline$\alpha_{\max }^{e}$ & $95 \%$ & 5567 & 3415 & 2607 & 2144 & 1674 & 1259 & 995 \\
$\alpha_{\max }^{e}$ & $99 \%$ & 4230 & 2595 & 1981 & 1629 & 1272 & 957 & 756 \\
\hline
\end{tabular}

Table III shows that for the EPFL $720 \mathrm{kVA} / 560 \mathrm{kWh}$ BESS and for the $T \leq 6 \mathrm{~h}$, the theoretical value of $\alpha_{\max }$ is determined by the BESS power ratings rather than its energy capability. In Section IV-C, details are given about the values of $T$ and $\alpha_{\max }$ used to experimentally validate the methods proposed in this paper with the specific ratings of the EPFL BESS.

\section{B. Simulations}

The performance of the proposed control framework is validated by simulations in Matlab/Simulink. The simulator uses one year of frequency measurements for the NGET database as

\begin{tabular}{|c|c|c|c|c|c|c|c|}
\hline$T[h]$ & 1 & 2 & 3 & 4 & 6 & 12 & 24 \\
\hline \multicolumn{8}{|c|}{ AR(0) model } \\
\hline$\alpha_{\max }^{e}$ & 14807 & 8524 & 6278 & 4959 & 3623 & 2270 & 1270 \\
\hline$\alpha_{\max }^{p}$ & 2200 & 2900 & 3133 & 3250 & 3366 & 3483 & 3541 \\
\hline \multicolumn{8}{|c|}{ AR(8) model } \\
\hline$\alpha_{\max }^{e}$ & 17216 & 9751 & 7337 & 5895 & 4302 & 2633 & 1386 \\
\hline$\alpha_{\max }^{p}$ & 2200 & 2900 & 3133 & 3250 & 3366 & 3483 & 3541 \\
\hline
\end{tabular}

TABLE III

DROOP COEFFICIENT VALUES $[k W / H z]$, EPFL DATABASE $(\rho=95 \%)$.

input and the BESS state of energy as output. It presents two different models of BESS. In the ideal model the losses are not taken into account, whereas in the real one the losses are modeled as in [7]. Specifically the battery is considered as the series of a voltage sources and a resistance and the values of both depend on the battery state of charge (SOC). The SOE-M performance in the simulation environment has been evaluated for three periods $T$, two different confidences levels $(95 \%$ and $99 \%)$, and for the two different autoregressive models $(A R(0)$ and $A R(8)$ ). The results of these simulations are evaluated by quantifying the failure rate $\lambda_{T}$. The SOE-M fails in its control when the BESS reaches its capability limits and cannot provide power for frequency regulation. The control failure rate $\lambda_{T}$ corresponds to the duration of the period in which the BESS is not able to perform its tasks, expressed in percentage of the simulation duration. From the definition of the droop coefficient given in Section III-A, over one period of operation the expected failure rate is related to the confidence $\rho$ on the control action as follows:

$$
\tilde{\lambda}_{T}=1-\rho .
$$

TABLE IV

Simulation RESUlts, NGET Database

\begin{tabular}{|l|cc|cc|cc|}
\multicolumn{4}{c}{} & \multicolumn{2}{c}{$A R(0)$} & \multicolumn{2}{c|}{$A R(8)$} \\
\hline BESS model & $\rho$ & $T$ & $\alpha_{\max }$ & $\tilde{\lambda}_{T}$ & $\alpha_{\max }$ & $\tilde{\lambda}_{T}$ \\
& {$[\%]$} & {$[h]$} & {$[k W / H z]$} & {$[\%]$} & {$[k W / H z]$} & {$[\%]$} \\
\hline Ideal & $95 \%$ & 24 & 893 & 3.631 & 995 & 4.723 \\
Real & $95 \%$ & 24 & 893 & 4.423 & 995 & 6.410 \\
Real & $99 \%$ & 24 & 679 & 0.358 & 755 & 0.358 \\
Ideal & $95 \%$ & 12 & 1201 & 4.087 & 1259 & 4.609 \\
Real & $95 \%$ & 12 & 1201 & 4.779 & 1259 & 5.223 \\
Real & $99 \%$ & 12 & 912 & 0.616 & 957 & 0.799 \\
Ideal & $95 \%$ & 1 & 5325 & 2.992 & 5567 & 2.472 \\
Real & $95 \%$ & 1 & 5325 & 5.086 & 5567 & 2.452 \\
Real & $99 \%$ & 1 & 4045 & 0.408 & 4229 & 0.350 \\
\hline
\end{tabular}

Table IV presents the results of the simulations. The failure rate $\lambda_{T}$ is on average equal to $3.8 \%$ for the simulation based 
on a $\rho=95 \%$ and a BESS with unitary efficiency and to $4.7 \%$ for the simulation based on a $\rho=95 \%$ and a BESS with nonunitary efficiency. In both cases the average failure rate for the simulation is close to the expected value of $5 \%$. Moreover, it can be observed that the introduction of a more accurate BESS efficiency model does not provide results considerably different from those obtained for an ideal BESS.

\section{Experiments}

The algorithm has been tested experimentally using a dedicated setup consisting in a grid-connected $720 \mathrm{kVA} / 560 \mathrm{kWh}$ BESS installed at the EPFL campus in Lausanne [7]. Frequency measurements are from an on-site PMU-based metering system [10]. Three sets of experiments have been carried out.

The purpose of the first group of experiments is the evaluation of the ability of the algorithm to ensure the rated reliability. Such series of experiments has been carried out for $T=3 \mathrm{~h}$ and $T=6 \mathrm{~h}$ and with the $\alpha_{\max }$ defined as in section III-A, based on the BESS energy capability. The choice of $T=3 \mathrm{~h}$ and $T=6 \mathrm{~h}$, although in contrast with the results presented in Table III, is justified by two observations. On one hand, experiments based on $T=12 \mathrm{~h}$ or $T=24 \mathrm{~h}$ would need a considerably long run-time to achieve statistically relevant results. On the other side, for smaller $T$, an $\alpha_{\max }$ computed as in Section III-A would generate an ineffective regulation, since even for deviations of relatively small magnitude, the BESS power would be limited by its power rating and the regulation signal would not be tracked accurately. For $T=3 \mathrm{~h}$ and $T=6 \mathrm{~h}$, the power rating of the battery would limit the BESS power from tracking the regulating signal only in the occurrence of large and infrequent frequency deviations $(\Delta f>100 \mathrm{mHz})$. These values of $T$ are therefore suited to the purpose of validating the proposed method, although the consideration of the power ratings should be taken into account in the sizing phase of a BESS designed to perform PFR.

Figure 5 shows the result of EXP5. The control fails every time the SOC is near to the limits and the battery is not able to implement the set points received from the control logic. The results of this first set of experiments are summarised in

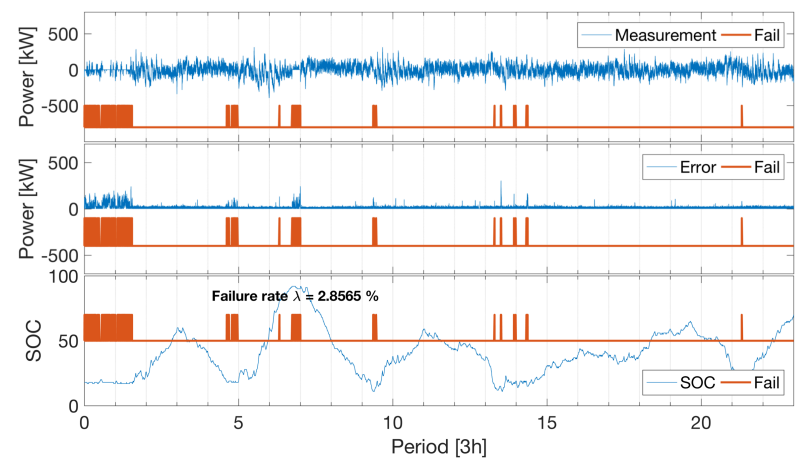

Figure 5. Evaluation of the reliability. Results from EXP5. Top: power output, middle: absolute error between setpoint and measurement, bottom: SOE.

Table $\mathrm{V}$ and consist of almost $500 \mathrm{~h}$ of PFR divided in four experiments with duration $H$ of 23 periods and 30 periods, respectively. The observed failure rates $\hat{\lambda}_{T}$ for each experiment are in the neighbourhood of the expected value $\tilde{\lambda}_{T}=5 \%$ with the experiment based on $A R(0)$ and $T=6 \mathrm{~h}$ being the one in which $\hat{\lambda}_{T}$ is the more distant from this value, having a $\widehat{\lambda}_{T}=2.86 \%$.

TABLE V

EXPERIMENTAL RESULTS: RELIABILITY AND FAILURE RATE.

\begin{tabular}{|c|ccccc|}
\hline Name & Model & $\begin{array}{c}T \\
{[h]}\end{array}$ & $\begin{array}{c}H \\
{[\text { periods }]}\end{array}$ & $\begin{array}{c}\alpha_{\max } \\
{[k W / H z]}\end{array}$ & $\begin{array}{c}\widehat{\lambda}_{T} \\
{[\%]}\end{array}$ \\
\hline EXP3 & AR8 & $3 \mathrm{~h}$ & 23 & 7337 & 5.9871 \\
EXP4 & AR0 & $3 \mathrm{~h}$ & 23 & 6278 & 5.7596 \\
EXP5 & AR0 & $6 \mathrm{~h}$ & 30 & 4302 & 2.8565 \\
EXP6 & AR8 & $6 \mathrm{~h}$ & 30 & 3623 & 5.5144 \\
\hline
\end{tabular}

The second group of experiments aims to asses the ability of the BESS to follow the regulating signal. With regard to this purpose, the performance metrics chosen are the ones adopted by PJM Interconnect [8]. Such parameters evaluate the regulating action for each hour of service giving a general score which is the mean of three parameters delay correlation and precision. Figure 6 shows the performance scores for the $560 \mathrm{kWh}$ BESS object of this study, obtained on an experiment with period $T=1 \mathrm{~h}$ and a droop coefficient $\alpha=2200 \mathrm{~kW} \mathrm{~Hz}^{-1}$ and with overall duration of about 40 hours. Similar values has been registered in all the experiments presented and are aligned with the expectations for the battery which are usually around $96-99 \%$ [13].

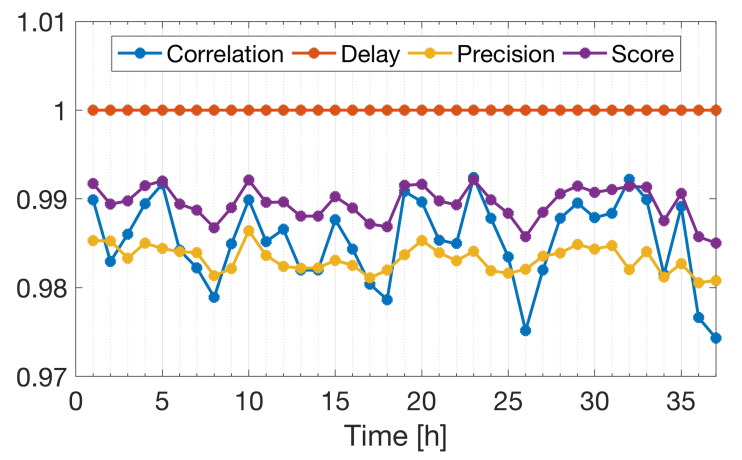

Figure 6. Performance assessment in providing frequency regulation services. Experimental results obtained for $T=1 \mathrm{~h}$ and $\alpha=2200 \mathrm{~kW} \mathrm{~Hz}^{-1}$.

The objective of the third group of experiments is gathering experimental data to playback in the simulator in order to validate its output and support the proposed simulation results. To quantify this, data from the experiments presented in Section IV-C has been fed as input for the simulator and the power and SOE profiles generated by the simulations have been compared to the experimental data. The accuracy of the simulation environment has been quantified via the RMS value of the difference between the measured and simulated SOE, $\triangle S O E^{R M S}$. Table VI shows the rms value of the 
TABLE VI

EXPERIMENTAL RESULTS: VALIDATION OF SIMULATION ENVIRONMENT.

\begin{tabular}{|c|ccccc|}
\hline Name & Model & $T$ & $H$ & $\alpha_{\max }$ & $\Delta S O E^{R M S}$ \\
& & {$[\mathrm{~h}]$} & {$[$ periods $]$} & {$[\mathrm{kW} / \mathrm{Hz}]$} & {$[-]$} \\
\hline EXP1 & AR0 & 1 & 39 & 2200 & 0.007 \\
EXP2.1 & AR8 & 1 & 24 & 2200 & 0.009 \\
EXP2.2 & AR8 & 1 & 42 & 2200 & 0.006 \\
EXP2.3 & AR8 & 1 & 30 & 2200 & 0.005 \\
EXP3 & AR8 & 3 & 23 & 7337 & 0.028 \\
EXP4 & AR8 & 3 & 23 & 6278 & 0.107 \\
EXP5 & AR0 & 6 & 30 & 4302 & 0.076 \\
\hline
\end{tabular}

difference between measured and simulated SOE for various experiments. This value is on average of 0.034 , indicating satisfactory simulation performances. Figure 7 shows the SOE profiles for one of such experiments. The simulated SOE matches closely the measured one, except for the moments when the control fails due to a SOE approaching its lower bound. This difference at extreme SOE values is due the fact that the BESS internal controller imposes SOE limits that are dynamic and not always corresponding to the static bounds used in the simulation tool. Nonetheless, good simulation performances are achieved for a wide SOE range.

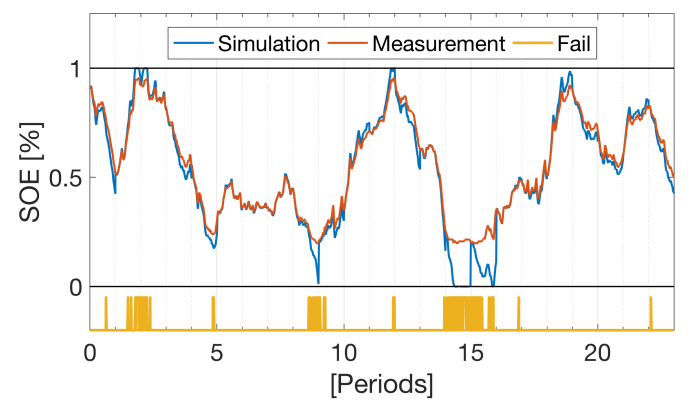

Figure 7. Performance assessment of the simulation tool, scenario from EXP3.

\section{CONCLUSIOns}

We present a droop-based control framework to perform primary frequency regulation by using a BESS. The computation of the droop coefficient and the state of energy management rely on the forecast of the energy needed for regulation over a multi-hour horizon, performed by means of autoregressive models. We show that, by exploiting the information provided by such forecasts, the BESS control can exploit a higher droop coefficient value (and thus the provide more regulating power), while ensuring the same level of reliability of a base case, $\operatorname{AR}(0)$, in which such information is not exploited. This constitutes an improvement over typical offset-based controls for BESSs providing frequency regulation (e.g. [5], [14]), although it requires large datasets of historical data (not always easily accessible) to train the autoregressive models.
The proposed method is both validated via simulations and implemented in the control of a grid-connected $720 \mathrm{kVA} / 560 \mathrm{kWh}$ Lithium titanate BESS. The experiments carried out in the present work demonstrate the effectiveness as well as the practical deployability of the proposed control framework. Moreover, we exploit experimental data to assess the performances of the BESS in providing primary frequency regulation, which prove to be extremely high.

Future works concern the improvement of the forecasting tools performance, the introduction of a period-based computation of the droop coefficient (e.g. definition of $\alpha$ on a weekly basis) and the coupling of the proposed optimal control scheme with an upper-level optimization problem to determine the optimal battery capacity.

\section{REFERENCES}

[1] A. Malhotra, B. Battke, M. Beuse, A. Stephan, and T. Schmidt, "Use cases for stationary battery technologies: A review of the literature and existing projects," Renewable and Sustainable Energy Reviews, vol. 56, pp. 705-721, 2016.

[2] H. Bevrani, Robust power system frequency control; 2nd ed., ser. Power Electronics and Power Systems. Dordrecht: Springer, 2014. [Online]. Available: http://cds.cern.ch/record/1967886

[3] Energy storage and storage services, ENTSO-E position. ENTSO-E, October 2016

[4] X. Lu, K. Sun, J. M. Guerrero, J. C. Vasquez, and L. Huang, "Stateof-charge balance using adaptive droop control for distributed energy storage systems in dc microgrid applications," IEEE Transactions on Industrial electronics, vol. 61, no. 6, pp. 2804-2815, 2014.

[5] O. Mégel, J. L. Mathieu, and G. Andersson, "Maximizing the potential of energy storage to provide fast frequency control," in Innovative Smart Grid Technologies Europe (ISGT EUROPE), 2013 4th IEEE/PES. IEEE, 2013, pp. 1-5.

[6] M. Khalid and A. V. Savkin, "Model predictive control based efficient operation of battery energy storage system for primary frequency control," in Control Automation Robotics \& Vision (ICARCV), 2010 11th International Conference on. IEEE, 2010, pp. 2248-2252.

[7] F. Sossan, E. Namor, R. Cherkaoui, and M. Paolone, "Achieving the dispatchability of distribution feeders through prosumers data driven forecasting and model predictive control of electrochemical storage," IEEE Transactions on Sustainable Energy, vol. 7, no. 4, pp. 1762-1777, Oct 2016.

[8] C. Pilong, PJM Manual 12: Balancing Operations, 36th ed., PJM, January 2017.

[9] B. Xu, A. Oudalov, J. Poland, A. Ulbig, and G. Andersson, "Bess control strategies for participating in grid frequency regulation," IFAC Proceedings Volumes, vol. 47, no. 3, pp. 4024-4029, 2014.

[10] M. Pignati, M. Popovic, S. Barreto, R. Cherkaoui, G. D. Flores, J.-Y. Le Boudec, M. Mohiuddin, M. Paolone, P. Romano, S. Sarri et al., "Real-time state estimation of the epfl-campus medium-voltage grid by using pmus," in Innovative Smart Grid Technologies Conference (ISGT), 2015 IEEE Power \& Energy Society. IEEE, 2015, pp. 1-5.

[11] H. Madsen, Time Series Analysis, ser. Chapman \& Hall/CRC Texts in Statistical Science. Taylor \& Francis, 2007.

[12] B. Xu, A. Oudalov, J. Poland, A. Ulbig, and G. Andersson, "Bess control strategies for participating in grid frequency regulation," IFAC Proceedings Volumes, vol. 47, no. 3, pp. 4024-4029, 2014.

[13] E. P. M. Co, "Final technical performance report: Grid-scale energy storage demonstration of ancillary services using the ultrabatteryr technology," East Penn Manufacturing Co, Tech. Rep. DE-OE0000302, August 2015.

[14] A. Oudalov, D. Chartouni, and C. Ohler, "Optimizing a battery energy storage system for primary frequency control," IEEE Transactions on Power Systems, vol. 22, no. 3, pp. 1259-1266, 2007. 